\title{
GMR
}

\section{Hypoxia-related gene expression in porcine skeletal muscle tissues at different altitude}

\author{
J. Zhang ${ }^{1 *}$, L. Chen ${ }^{2 *}$, K.R. Long ${ }^{2}$ and Z.P. $\mathrm{Mu}^{3}$ \\ ${ }^{1}$ Department of Animal Science, \\ Southwest University at Rongchang, Chongqing, China \\ ${ }^{2}$ Institute of Animal Genetics and Breeding, \\ College of Animal Science and Technology, \\ Sichuan Agricultural University, Ya'an, Sichuan, China \\ ${ }^{3}$ Department of Animal Science and Technology, \\ Chongqing Three Gorges Vocational College, Chongqing, China \\ *These authors contributed equally to this study. \\ Corresponding author: J. Zhang \\ E-mail: zhangjie813@163.com
}

Genet. Mol. Res. 14 (3): 11587-11593 (2015)

Received February 2, 2015

Accepted May 26, 2015

Published September 28, 2015

DOI http://dx.doi.org/10.4238/2015.September.28.10

\begin{abstract}
Hypoxia influences many physiological processes, such as respiration, cardiovascular, neurophysiology, and digestion. Skeletal muscle is an important motor organ, which relies on oxygen of oxidation; however, the study of hypoxia in skeletal muscle is lacking. In order to understand the effect of hypoxia on skeletal muscle, we determined the expression level of four hypoxia-related genes (ADAM17, ARG2, MMP, and HIF1A) in two distinct skeletal muscle tissues from Tibetan pigs that live at different altitudes (500 and 3650 $\mathrm{m})$. Consistent with the well-characterized role of four hypoxia-related genes in the adaptation to high altitude, we found that, compared with the plain pigs, the plateau pigs had higher mRNA abundances of the four genes and lower myofiber ratio in skeletal muscle. The negative correlation between the myofiber ratio and mRNA abundance of the four hypoxia-related genes highlights their critical roles in skeletal
\end{abstract}


muscle. These findings may be important for understanding skeletal muscle adaptation to high altitude and hypoxia-related muscle diseases in humans.

Key words: Hypoxia; Gene expression; Pig; Muscle

\section{INTRODUCTION}

In geology and earth science, a plateau, which is also called a high plain or tableland, is an area of highland that usually consists of relatively flat terrain. As it is well-known, the plateau is characterized by hypoxia, cold temperatures, intense radiation, low humidity, infrabar, and other environment conditions. As an extreme environment, the plateau forms unique geomorphy, and is home to unique species of animals, plants, and of human populations. There are many famous plateaus in the world, such as the Qinghai-Tibet Plateau, Pamirs Plateau, Brazilian Plateau, and Mexican Plateau. The Qinghai-Tibetan Plateau is the largest continuous high elevation ecosystem in the world with an average elevation of more than $4000 \mathrm{~m}$ (Yang et al., 2011a). The Qinghai-Tibetan Plateau is a unique geomorphic unit that covers an area of $2572.4 \times 10^{3} \mathrm{~km}^{2}$, and occupies $26.8 \%$ of the land of China (Zhang et al., 2002) and $1 \%$ of the terrene (Wu et al., 2001). The abundant species (i.e., Tibetan pig, Tibetan chicken, Tibetan goat, and yak) that formed under the unique geographical conditions of the Qinghai-Tibetan Plateau provide perfect model organisms for high-altitude medicine research.

Tibetan pigs are a unique, local, feral subspecies of domestic pig that inhabit the Qinghai-Tibet plateau and are mainly distributed in the Tibetan highlands (Yang et al., 2011b). Tibetan pigs show striking phenotypic and physiological differences from lowland pigs (strong adaptability, robustness and resistance, thin skin, high meat cutability, extra-fine muscle fibers, more intramuscular fat, tasty meat, strong wild flavor, low reproductive rate, and slow growth) (Zhu et al., 2009), which have allowed them to become well-adapted to the extreme conditions, such as hypoxia. Hypoxia is a main feature of plateaus and has a diverse influence on biota. For example, hypoxic mice develop severe pulmonary hypertension (right ventricular systolic pressure $60 \mathrm{mmHg}$ ) as compared with normoxic mice $(27 \mathrm{mmHg}$ ) (Fagan et al., 2001). In addition, pig are devoid of brown fat post natally and have similar metabolic features, cardiovascular systems, and proportional organ sizes as human (Spurlock and Gabler, 2008), making the Tibetan pig an excellent model for high altitude medicine research.

Skeletal muscle comprises $\sim 40$ to $45 \%$ of the total mass and $\sim 60 \%$ of the total body protein in humans (Wagenmakers, 1998). It is of obvious importance for locomotion and is one of the most metabolically active tissues in the body (Gasier et al., 2010). Active skeletal muscle requires oxygen for oxidation to provide energy, indicating the importance of the atmospheric oxygen content in skeletal muscle. Previous studies have reported that the expression of some genes changes under hypoxic conditions compared with normoxic conditions, which induces a series of physiological variation (van der Meer et al., 2005; Fu et al., 2010). However, little is known about the effect of hypoxia on skeletal muscle tissues.

Here, we measured the myofiber ratio and the expression of four hypoxia-related genes across two phenotypically distinct skeletal muscles in Tibetan pigs from different altitudes. This study will aid a better understanding of the molecular mechanisms of skeletal muscle adaptation to high altitude. 


\section{MATERIAL AND METHODS}

\section{Tissue collection and measurement}

Nine female Tibetan pigs from Chengdu plain (CTP: about $500 \mathrm{~m}$ altitude) and nine from Rikaze (RTP: about $3650 \mathrm{~m}$ altitude) were used in this study. Animals were humanely sacrificed at 180 days old, in compliance with the guidelines for the Care and Use of experimental animals established by the Ministry of Agriculture of China. The longissimus dorsi muscle $(\mathrm{LDM})$ and psoas major muscle (PMM) were dissected immediately after sacrifice, frozen in liquid nitrogen, and stored at $-80^{\circ} \mathrm{C}$. The myofiber ratio (type I vs II) was measured for each muscle tissue as described previously (Lefaucheur et al., 2002).

\section{Quantitative PCR (Q-PCR)}

Total RNA was extracted from the skeletal muscles using TRIzol regent (Invitrogen, Carlsbad, CA, USA) according to the manufacturer protocol. Total RNA was reverse transcribed to cDNA using the oligo (dT) and random 6-mers primers provided in the PrimeScript RT Master Mix kit (TaKaRa, Dalian, China), following the manufacturer protocol. Q-PCR was performed using the SYBR Premix Ex Taq kit (TaKaRa, Dalian, China) on a CFX96 RealTime PCR detection system (Bio-Rad, Richmond, CA, USA). Primer sequences used for Q-PCR analysis are shown in Table 1. All experiments contained a negative control and all Q-PCR reactions were performed in triplicate.

\begin{tabular}{|c|c|c|c|}
\hline Gene symbol & Primer sequences (5' to $3^{\prime}$ ) & Amplicon size (bp) & Ensembl ID \\
\hline$A C T B^{*}$ & $\begin{array}{l}\text { F: TCTGGCACCACACCTTCT } \\
\text { R: TGATCTGGGTCATCTTCTCAC }\end{array}$ & 114 & ENSSSCG00000007585 \\
\hline TBP* & $\begin{array}{l}\text { F: GATGGACGTTCGGTTTAGG } \\
\text { R: AGCAGCACAGTACGAGCAA }\end{array}$ & 124 & ENSSSCG00000022683 \\
\hline TOP $2 B^{*}$ & $\begin{array}{l}\text { F: AACTGGATGATGCTAATGATGCT } \\
\text { R: TGGAAAAACTCCGTATCTGTCTC }\end{array}$ & 137 & ENSSSCG00000011213 \\
\hline$A R G 2$ & $\begin{array}{l}\text { F: CGCGAGTGCATTCCATCCT } \\
\text { R: TCCAAAGTCTTTTAGGTGGCAG }\end{array}$ & 170 & ENSSSCG00000002294 \\
\hline ADAM17 & $\begin{array}{l}\text { F: GACTCTAGGGTTCTAGCCCAC } \\
\text { R: GGAGACTGCAAACGTGAAACAT }\end{array}$ & 173 & ENSSSCG00000008641 \\
\hline$M M P 3$ & $\begin{array}{l}\text { F: CGGTTCCGCCTGTCTCAAG } \\
\text { R: CGCCAAAAGTGCCTGTCTT }\end{array}$ & 206 & ENSSSCG00000014986 \\
\hline HIFIA & $\begin{array}{l}\text { F: GAACGTCGAAAAGAAAAGTCTCG } \\
\text { R: CCTTATCAAGATGCGAACTCACA }\end{array}$ & 124 & ENSSSCG00000005096 \\
\hline
\end{tabular}

*ACTB ( $\beta$ actin), TBP (TATA box binding protein), and TOP2B (topoisomerase II $\beta$ ) are the endogenous control genes. $\mathrm{F}$ indicates forward primers, and $\mathrm{R}$ indicates reverse primers.

\section{Statistical analysis}

The relative expression of mRNA was calculated using the $2^{-\Delta \Delta \mathrm{Ct}}$ method. All statistical analyses were conducted with the SPSS Statistics 21.0 software (IBM, New York, NY, USA). The significance of differences was determined by the Student $t$-test. Data are reported as means \pm standard deviation. 


\section{RESULTS AND DISCUSSION}

\section{Analysis of myofiber ratio}

Compared with the RTPs, the CTPs showed a higher myofiber ratio (type I vs II) (Student $t$-test, $\mathrm{P}<0.01$; Figure 1) in LDM and PMM, which is consistent with environment selection. The RTPs live in the wild, and need more energy to move in a fast and powerful manner to escape from predators and to chase and catch prey than the CTPs do, which live under temperate conditions without threat from enemy and with food. This adaption to life in the wild might be reflected in the myofiber composition of the skeletal muscle, such that a higher proportion of type II fibers are needed. This is in accordance with the functional differences of type I and II myofibers; type I myofiber-rich muscle is highly oxidative and is adapted to perform for long durations and has low energy consumption (Janda, 1983). Conversely, muscles with a higher proportion of type II myofibers are better adapted for movement $(\mathrm{Ng}$ et al., 1998). In addition, the air in Chengdu Plain had higher oxygen content than the air at Rikaze, highlighting distinct differences in oxygen use by muscle tissue. A previous study demonstrated that high-altitude hypoxia in human subjects increased the activity of oxidative enzymes (Terrados, 1992) and inhibited the normal shift from type II to type I fibers, which occurs during postnatal development in the rat soleus muscle (Ishihara et al., 1995; Itoh et al., 1995). This means that there is a lower myofiber ratio (type I vs II) under hypoxic conditions compared with that observed under normoxia, which is similar to the results observed with LDM and PMM.

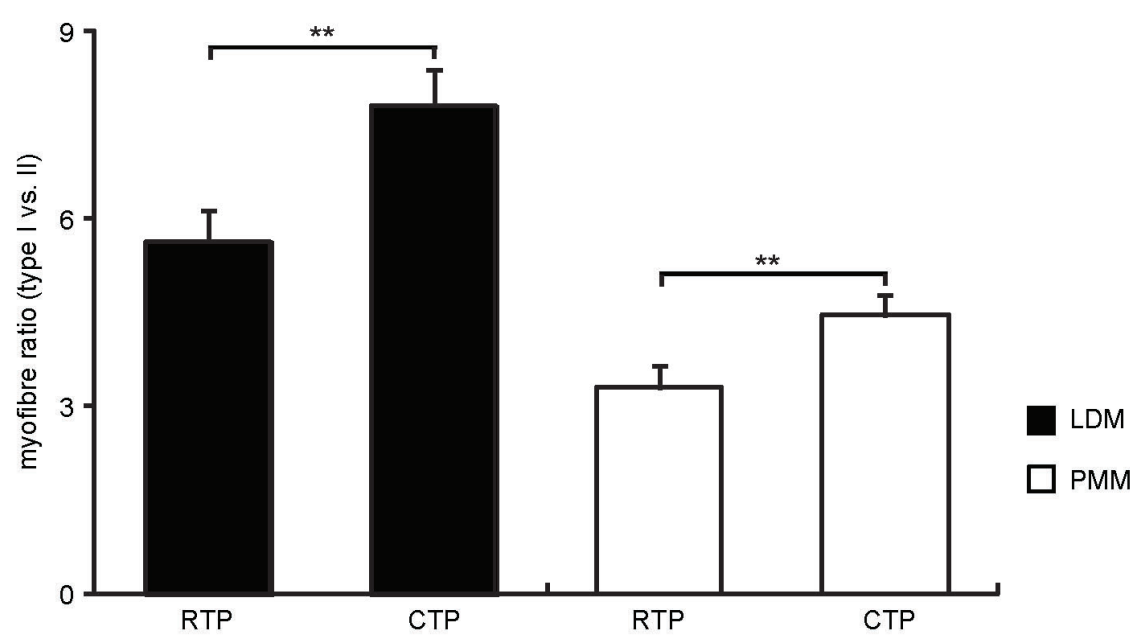

Figure 1. Different myofiber ratios (type I $v s$ II) between samples. Data are reported as means $\pm \mathrm{SD}$. The significance of differences between samples was determined by the Student $t$-test; RTP, CTP, LDM, and PMM refer to the Rikaze Tibetan pig, Chengdu Tibetan pig, longissimus dorsi muscle, and psoas major muscle tissue, respectively. $* * \mathrm{P}<0.01$.

\section{Analysis of hypoxia-related gene expression}

We investigated the expression of four genes in response to functional hypoxia: ADAM metallopeptidase domain 17 (ADAM17), arginase 2 (ARG2), matrix metallopeptidase 
3 (MMP3), and hypoxia-inducible factor 1A (HIF1A). In the LDM and PMM, mRNA levels of the four hypoxia-related genes were significantly higher in RTP than in CTP (Student $t$-test, $\mathrm{P}<0.01$; Figure 2). In the yak, ADAM17 is a candidate gene associated with the HIF pathway, which plays a crucial role in the response to hypoxia (Qiu et al., 2012). Simonson et al. (2010) showed that alleles of human $A D A M 17$ were present at significantly different frequencies in Tibetans and low-altitude dwellers. The $A R G 2$ and $M M P 3$ genes are involved in the hypoxia response, and underwent positive selection in yak compared to none in cattle (Qiu et al., 2012). Our study also demonstrates that the RTPs have significantly higher expression of the $A R G 2$ and MMP3 genes in LDM and PMM than the CTPs do. As a master regulator of the cellular response to hypoxia, HIF $1 A$ triggers the widespread transcription of genes involved in numerous physiological processes (Sogawa et al., 1998; Yee Koh et al., 2008). For instance, upregulation of $H I F 1 A$, possibly as part of a larger inflammatory response mediated by $T N F-\alpha$, may also contribute to resistance versus susceptibility to hypoxic conditions (Woelk et al., 2012). HIF1A can induce expression of the WASF3 gene, which is an important mediator of cell motility, invasion, and metastasis under hypoxic conditions (Ghoshal et al., 2012).

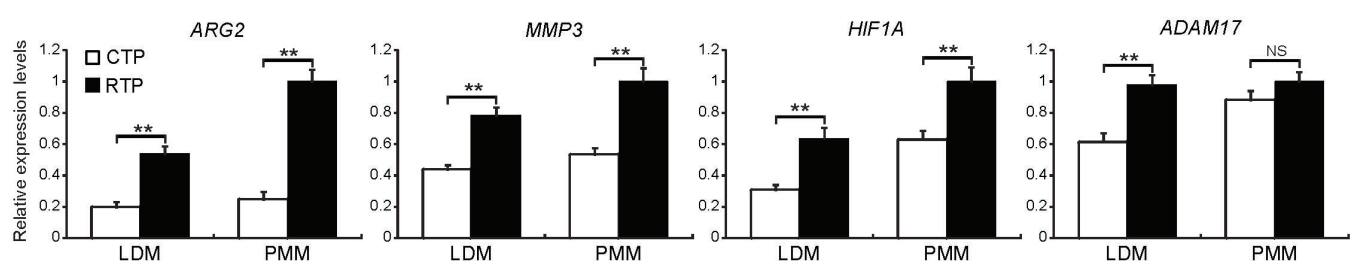

Figure 2. Relative expression levels of four hypoxia-related genes. The significance of differences between samples was determined by the Student $t$-test; RTP, CTP, LDM, and PMM refer to the Rikaze Tibetan pig, Chengdu Tibetan pig, longissimus dorsi muscle, and psoas major muscle tissue, respectively. ${ }^{*} \mathrm{P}<0.01$; ${ }^{\mathrm{NS}}$ not significant $(\mathrm{P}>0.05)$.

\section{Correlation between myofiber ratio and hypoxia-related genes}

The mRNA levels of ADAM17 $(r=-0.17, \mathrm{P}=0.31), A R G 2(r=-0.38, \mathrm{P}=0.02)$, HIF1A $\left(r=-0.61, \mathrm{P}=7.55 \times 10^{-5}\right)$, and $M M P 3\left(r=-0.66, \mathrm{P}=9.39 \times 10^{-6}\right)$ were negatively correlated with the myofiber ratio (Figure 3), highlighting the critical roles of these genes in skeletal muscle adaptation to high altitude.

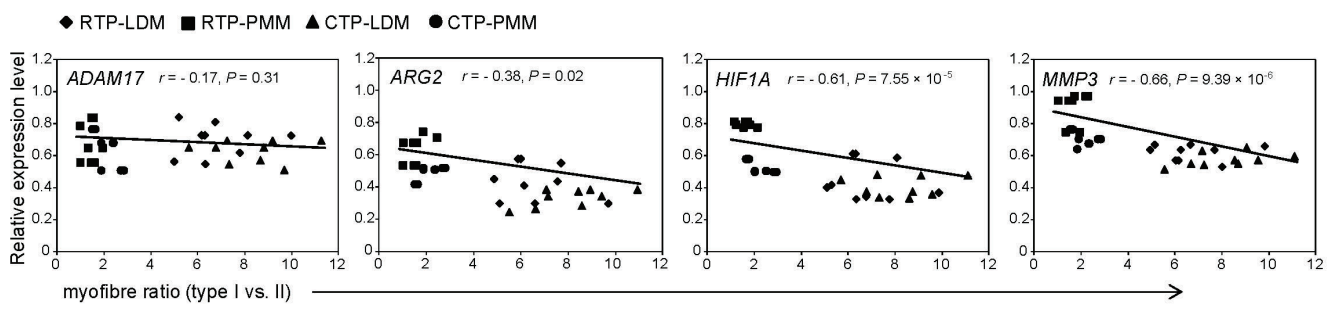

Figure 3. Pearson's correlation test comparing the myofiber ratio and the mRNA abundance of four hypoxia-related genes. RTP, CTP, LDM, and PMM refer to the Rikaze Tibetan pig, Chengdu Tibetan pig, longissimus dorsi muscle, and psoas major muscle tissue, respectively. 


\section{CONCLUSION}

In summary, these results are consistent with analyses of hypoxia related genes in other species (i.e., yak). In addition to playing a key role in the adaptation of metabolism in pigs at high altitude, we propose that $A D A M 17, A R G 2, M M P$, and $H I F 1 A$ genes may acts as candidate genes for high altitude medicine research.

\section{Conflicts of interest}

The authors have no conflict of interest to declare.

\section{ACKNOWLEDGMENTS}

Research supported by grants from the Fundamental Research Funds for the Central Universities (\#SWU114059).

\section{REFERENCES}

Fagan KA, Morrissey B, Fouty BW, Sato K, et al. (2001). Upregulation of nitric oxide synthase in mice with severe hypoxia-induced pulmonary hypertension. Respir. Res. 2: 306-313.

Fu H, Luo F, Yang L, Wu W, et al. (2010). Hypoxia stimulates the expression of macrophage migration inhibitory factor in human vascular smooth muscle cells via HIF-1 $\alpha$ dependent pathway. BMC Cell Biol. 11: 66.

Gasier HG, Fluckey JD and Previs SF (2010). The application of 2H2O to measure skeletal muscle protein synthesis. Nutr. Metab. 7: 31 .

Ghoshal P, Teng Y, Lesoon L and Cowell JK (2012). HIF1A induces expression of the WASF3 metastasis associated gene under hypoxic conditions. Int. J. Cancer 131: E905-E915.

Ishihara A, Itoh K, Oishi Y, Itoh M, et al. (1995). Effects of hypobaric hypoxia on histochemical fibre-type composition and myosin heavy chain isoform component in the rat soleus muscle. Pflugers Arch. 429: 601-606.

Itoh K, Itoh M, Ishihara A, Hirofuji C, et al. (1995). Influence of 12 weeks of hypobaric hypoxia on fibre type composition of the rat soleus muscle. Acta Physiol. Scand. 154: 417-418.

Janda V (1983). On the concept of postural muscles and posture in man. Aust. J. Physiother. 29: 83-84.

Lefaucheur L, Ecolan P, Plantard L and Gueguen N (2002). New insights into muscle fiber types in the pig. J. Histochem. Cytochem. 50: 719-730.

Ng JK-F, Richardson CA, Kippers V and Parnianpour M (1998). Relationship between muscle fiber composition and functional capacity of back muscles in healthy subjects and patients with back pain. J. Orthop. Sports Phys. Ther. 27: 389-402.

Qiu Q, Zhang G, Ma T, Qian W, et al. (2012). The yak genome and adaptation to life at high altitude. Nat. Genet. 44: 946-949.

Simonson TS, Yang Y, Huff CD, Yun H, et al. (2010). Genetic evidence for high-altitude adaptation in Tibet. Science 329: $72-75$.

Sogawa K, Numayama-Tsuruta K, Ema M, Abe M, et al. (1998). Inhibition of hypoxia-inducible factor 1 activity by nitric oxide donors in hypoxia. Proc. Nat. Acad. Sci. U. S. A. 95: 7368-7373.

Spurlock ME and Gabler NK (2008). The development of porcine models of obesity and the metabolic syndrome. J. Nutr. 138: $397-402$.

Terrados N (1992). Altitude training and muscular metabolism. Int. J. Sports Med. 13 (Suppl 1): S206-209.

van der Meer DL, van den Thillart GE, Witte F, de Bakker MA, et al. (2005). Gene expression profiling of the long-term adaptive response to hypoxia in the gills of adult zebrafish. Am. J. Physiol. Regul. Integr. Comp. Physiol. 289: R1512-R1519.

Wagenmakers AJ (1998). Muscle amino acid metabolism at rest and during exercise: role in human physiology and metabolism. Exerc. Sport Sci. Rev. 26: 287-314.

Woelk CH, Zhang JX, Walls L, Viriyakosol S, et al. (2012). Factors regulated by interferon gamma and hypoxia-inducible factor 1A contribute to responses that protect mice from Coccidioides immitis infection. BMC Microbiol. 12: 218. 
Wu Y, Cui Z, Liu G, Ge D, et al. (2001). Quaternary geomorphological evolution of the Kunlun Pass area and uplift of the Qinghai-Xizang (Tibet) Plateau. Geomorphology 36: 203-216.

Yang S, Zhang H, Mao H, Yan D, et al. (2011a). The local origin of the Tibetan pig and additional insights into the origin of Asian pigs. PLoS One 6: e28215.

Yang S, Zhang H, Mao H, Yan D, et al. (2011b). The local origin of the Tibetan pig and additional insights into the origin of Asian pigs. PLoS One 6: e28215.

Yee Koh M, Spivak-Kroizman TR and Powis G (2008). HIF-1 regulation: not so easy come, easy go. Trends Biochem. Sci. 33: 526-534.

Zhang Y, Li B and Zheng D (2002). A discussion on the boundary and area of the Tibetan Plateau in China. Geogr. Res. 21: 1-8.

Zhu L, Li M, Li X, Shuai S, et al. (2009). Distinct expression patterns of genes associated with muscle growth and adipose deposition in tibetan pigs: a possible adaptive mechanism for high altitude conditions. High Alt. Med. Biol. 10: 45-55. 